\title{
Parameter Estimation and Prediction of a Nonlinear Storage Model: an algebraic approach
}

\author{
T.G. Doeswijk and K.J. Keesman
}

\begin{abstract}
Generally, parameters that are nonlinear in system models are estimated by nonlinear least-squares optimization algorithms. In this paper, if a nonlinear discrete-time model with a polynomial quotient structure in input, output, and parameters, a method is proposed to re-parameterize the model such that the model becomes linear in its new parameters. The new parameters can then be estimated by ordinary least squares. Finally, the model is rewritten in predictor form. A model of an agricultural storage facility with real data is presented to demonstrate the procedure and show the improved predictive performance. Some technical problems are indicated and solutions are proposed.
\end{abstract}

\section{INTRODUCTION}

In general, the model structure is an approximate representation of e.g. a specific physical process. Hence, there is a need to fit the model to the experimental data by parameter estimation. More specifically, as many physical models of real processes are nonlinear in the parameters, the model is fitted by nonlinear parameter estimation. In many applications, such as in optimal control studies, predictive quality is very important. Hence, the model with the estimated parameters should be validated on a different data set.

In literature various parameter estimation methods have been proposed. Among them the ordinary least-squares estimator, using matrix calculus, (see e.g. [1] and [2]) is most frequently used. Various extensions of this method have been proposed in the past. For instance, for solving nonlinear least-squares problems, different numerical optimizationbased procedures are available, see [3]. The key problems encountered in nonlinear least-squares estimation, especially in non-convex optimization problems, are the existence of local minima and the limited amount of parameters that can be reasonably estimated. Another extension is recursive leastsquares estimation, where the parameters are updated every time instant new data becomes available. This procedure can be extended to the nonlinear parameter case, see e.g. [4], as well.

The objective of this paper is to show how to estimate parameters in a nonlinear discrete-time model structure using ordinary least-squares techniques. Next to this, the predictive quality of the obtained reparameterized model is shown.

Manuscript received December 28, 2004. This work was financially supported with EET-subsidy from the Dutch Ministry of Finance, the Ministry of Education, Culture and Science and the Ministry of Housing, Regional Development and Environment (project no. EETK01120)

T.G. Doeswijk and K.J. Keesman are with the systems \& control group, Wageningen University, P.O. Box 17, 6700 AA Wageningen, The Netherlands (Phone: +31 317 483780, Fax: +31 317 484957, e-mail: karel. keesmandwur.nl)
Therefor, a method, together with the corresponding conditions on the model structure, is presented to reparameterize models that are nonlinear in their parameters as models linear in their parameters. The parameters of the reparameterized model can then be estimated by ordinary least-squares. Finally, the original model structure is retained but with the new parameters. The method is tested on data and a model of a storage facility. Basically, this paper has been inspired by the work of Ljung and Glad [5] and it is a natural extension of the paper by Lukasse et al. [6].

In section II the general derivation from a model with a polynomial quotient structure to a model linear in its parameters is presented and demonstrated by some examples. Section III shows three least-squares estimation techniques to estimate the parameters of a storage model with real data. Some validation results on the storage model are shown in section IV. In section $\mathrm{V}$ the estimation results as well as the applicability of the method are discussed. Finally, some conclusions are drawn in section VI.

\section{Modelling}

Physical modelling can generate equations that are not only nonlinear in their states and inputs but also in their parameters. If these nonlinear functions can be rearranged and reparameterized such that a function arises that is linear in its new parameters, these parameters can be directly and uniquely estimated using an ordinary least-squares procedure. After rearranging, the reparameterized model can be put in a predictor form. For a discrete-time model this is formally given in the following theorem:

Theorem 1: Given the discrete-time nonlinear model

$$
x_{k}=f(Z, p)
$$

where $Z=\left(x_{k-1}, \cdots, x_{k-\tau}, u_{k-1}, \cdots, u_{k-\tau}\right) \quad k, \tau \in \mathbb{Z}^{+}$ and $\tau<k$ with $\tau$ the time delay. If $f(\cdot)$ is a finite polynomial quotient in the elements of $\mathrm{Z}$ and $p$, the predictor

$$
\hat{x}_{k}=\tilde{f}(Z, \hat{\theta})
$$

is equivalent to (1). The unique ordinary least-squares estimate $\hat{\theta}$ is given by

$$
\hat{\theta}=\left(F(\cdot)^{T} F(\cdot)\right)^{-1} F(\cdot)^{T} F_{0}(\cdot)
$$

provided $\left(F(\cdot)^{T} F(\cdot)\right)^{-1}$ exists and where $F(\cdot)=$ $\left[F_{1}\left(x_{k}, Z\right) \ldots F_{n}\left(x_{k}, Z\right)\right]$ and $F_{0}(\cdot)=F_{0}\left(x_{k}, Z\right)$.

Proof: If $f(\cdot)$ is a finite polynomial quotient in $Z$ and $p$, equation (1) can be written as

$$
x_{k}=\frac{g(Z, p)}{h(Z, p)}
$$


with $g(\cdot)$ and $h(\cdot) \quad$ nite polynomials in elements of $Z$ and $p$. Then, multiplying both sides with $h(\cdot)$ and rearranging terms by elementary algebraic operations, as addition, subtraction, multiplication and division, results in

$$
\begin{aligned}
F_{0}\left(x_{k}, Z\right) & =F_{1}\left(x_{k}, Z\right) \theta_{1}+\ldots+F_{n}\left(x_{k}, Z\right) \theta_{n} \\
& =\left[\begin{array}{llll}
F_{1}(\cdot) & F_{2}(\cdot) & \ldots & F_{n}(\cdot)
\end{array}\right] \theta
\end{aligned}
$$

with, for $i=1, \ldots, n$ : $\theta_{i}=\varphi_{i}(p)$, a polynomial quotient in $p$. A unique least-squares estimate $\hat{\theta}$, given by (3), exists and is equal to $\theta$, if $F(\cdot)$ has rank $n$ and thus $\left(F(\cdot)^{T} F(\cdot)\right)^{-1}$ exists. Since $F_{0}\left(x_{k}, Z\right), \ldots, F_{n}\left(x_{k}, Z\right)$ are again nite polynomial quotients, equation (5) can be rearranged such that $\hat{x}_{k}=\tilde{f}(Z, \hat{\theta})=\tilde{f}(Z, \theta)$ with $\tilde{f}(\cdot)$ a nite polynomial quotient. After re-substitution using $\theta_{i}=\varphi_{i}(p)$ and rearranging terms: $\hat{x}_{k}=\tilde{f}(Z, \theta)=f(Z, p)$

Remark 1: Note that the model (1) is noise free. Then the estimate $\hat{\theta}$ is optimal, i.e. $\hat{\theta}=\theta$, which does not always hold for $p$ using nonlinear least squares as will be demonstrated in example 1. However, if noise is present the estimate is only unbiased if the noise sequence $\{e\}$ in $F_{0}(\cdot)=F(\cdot) \theta+e$, is uncorrelated with $F(\cdot)$ and has zero mean [2].

Remark 2: The reparameterized model (4) can also be put in an output error form by adding the output equation

$$
y_{k}=x_{k}+e_{k}
$$

The ordinary least squares estimate is then no longer optimal and the estimate may be biased. However, the estimate could be optimal in the sense that the predictor has a minimal mean square error over a validation data-set.

Example 1: Let us consider substrate consumption with Michaelis-Menten kinetics in a batch reactor. The substrate concentration is described by the following discrete-time model

$$
S_{k}=S_{k-1}-V_{\max } \frac{S_{k-1}}{K_{m}+S_{k-1}}
$$

By rearranging (7) we get

$$
S_{k-1}\left(S_{k}-S_{k-1}\right)=\left[-\left(\begin{array}{ll}
\left.S_{k}-S_{k-1}\right) & -S_{k-1}
\end{array}\right]\left[\begin{array}{c}
K_{m} \\
V_{\max }
\end{array}\right]\right.
$$

which is of the form $F_{0}(\cdot)=F(\cdot) \theta$ that can be solved by ordinary least-squares. Note that in this case $\theta=p$. The validity of the procedure can further be veri ed by generating model outputs with $S_{0}=30, K_{\mathrm{m}}=1$, and $V_{\max }=2$. A least-squares estimation using (8) gives $\hat{\theta}=\left[\begin{array}{ll}1 & 2\end{array}\right]^{T}$

In contrast to linear estimation, the outcome of a nonlinear parameter estimation procedure is not guaranteed as can be seen in Fig. 1. If no physical insight in terms of bounds is used and the estimation procedure is treated as a tting problem one could easily end up in a local minimum. But also with knowledge about system or parameter bounds care must be taken and the solution due to possible singularities in (7) is not always obvious.

Corollary 2: If, after rearranging (1), in (5): $F_{0}(\cdot)=0$, then a solution can be obtained from

$$
\hat{\theta}=\operatorname{ker}\left[F_{1}(\cdot) \cdots F_{n}(\cdot)\right]
$$

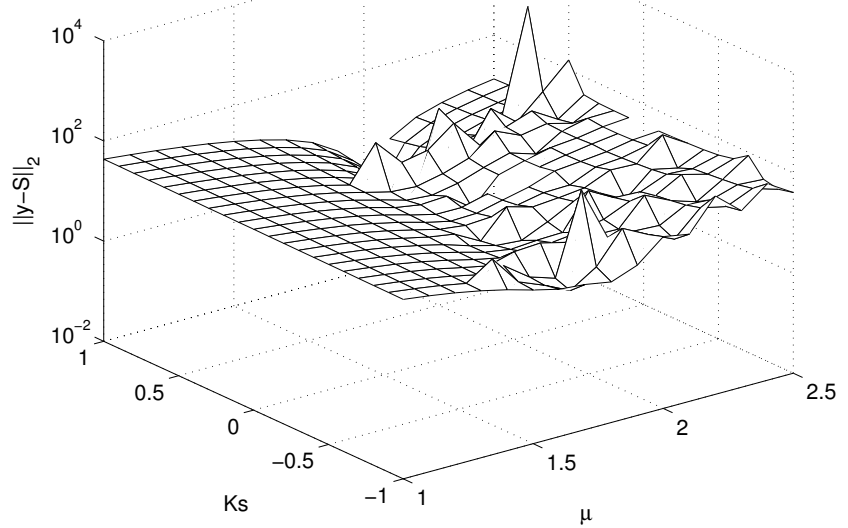

Fig. 1. Norm of the prediction error with $K_{m}=1, V_{\max }=2$.

In general, the non-empty solutions are not unique. The number of normalized solutions (nullity $(F)$ ) can be obtained from the well-known rank-nullity theorem [7]

$$
\operatorname{rank}(F)+\operatorname{nullity}(F)=n
$$

Example 2: Given the discrete-time system

$$
y_{k}=y_{k-1}-\frac{a}{b u_{k-1}+c}
$$

it can be rearranged as:

$$
0=\left[\begin{array}{ll}
1 & u_{k-1}\left(y_{k}-y_{k-1}\right) \\
y_{k}-y_{k-1}
\end{array}\right]\left[\begin{array}{lll}
a & b & c
\end{array}\right]^{T}
$$

and the solution is found by:

$$
\left[\begin{array}{lll}
\hat{a} & \hat{b} & \hat{c}
\end{array}\right]^{T}=\operatorname{ker}\left(\left[\begin{array}{ll}
1 & u_{k-1}\left(y_{k}-y_{k-1}\right) \\
y_{k}-y_{k-1}
\end{array}\right]\right)
$$

Now, with $y_{0}=10, u=\left[\begin{array}{llll}0 & 1 & 2 & 3\end{array}\right]^{T}$ and $a=1, b=$ $2, c=3: y=\left[\begin{array}{llll}10 & 9.67 & 9.47 & 9.33\end{array}\right]^{T}$ has been generated. Evaluating (13) gives the normalized solution: $\left[\begin{array}{lll}\hat{a} & \hat{b} & \hat{c}\end{array}\right]^{T}=$ $\left[\begin{array}{lll}0.27 & 0.53 & 0.80\end{array}\right]^{T} \simeq 0.27\left[\begin{array}{lll}a & b & c\end{array}\right]^{T}$, indicating that the system is unidentifi able.

Let us now apply the previous theory to a real-world application, i.e. storage of agricultural produce in a storage facility.

A discrete-time nonlinear model describing the temperature of the produce in a storage facility (see also [8]) is given by:

$$
\begin{aligned}
T_{p, k} & =\left(p_{1}+\frac{p_{2}}{p_{3}+p_{4} u_{k-1}}+\frac{p_{5}}{p_{6}+p_{7} u_{k-1}}\right) T_{p, k-1} \\
& +\frac{p_{8}+p_{9} u_{k-1}}{p_{3}+p_{4} u_{k-1}} T_{e, k-1}+\frac{p_{10}+p_{11} u_{k-1}}{p_{6}+p_{7} u_{k-1}} X_{e, k-1} \\
& +\left(p_{12}+\frac{p_{13}}{p_{6}+p_{7} u_{k-1}}\right)
\end{aligned}
$$

where $T_{p}$ is the product temperature, $T_{e}$ and $X_{e}$ the external temperature and absolute humidity respectively, $u$ the controlled input, i.e. product of ventilation and valve 
opening, and $p_{1} \ldots p_{13}$ are functions of physical and design parameters. Equation (14) can now be written as

$$
\begin{aligned}
& T_{p, k}=\left[\begin{array}{lll}
u_{k-1} T_{p, k} & u_{k-1}^{2} T_{p, k} & T_{p, k-1}
\end{array}\right. \\
& u_{k-1} T_{p, k-1} \quad u_{k-1}^{2} T_{p, k-1} \quad T_{e, k-1} \\
& u_{k-1} T_{e, k-1} \quad u_{k-1}^{2} T_{e, k-1} \quad X_{e, k-1} \\
& u_{k-1} X_{e, k-1} \quad u_{k-1}^{2} X_{e, k-1} \quad u_{k-1} \\
& \left.u_{k-1}^{2} 1\right]\left[\theta_{1} \cdots \theta_{14}\right]^{T}
\end{aligned}
$$

where $\theta_{i}=\varphi_{i}\left(p_{1}, \cdots, p_{13}\right)$, a polynomial quotient. Note that after reparameterization, $F_{0}(\cdot) \neq 0$ in (5).

Once the least-squares estimate $\hat{\theta}$ has become available the product temperature can be predicted by

$$
\hat{T}_{p, k}=\frac{\tilde{f}\left(T_{p, k-1}, T_{e, k-1}, X_{e, k-1}, u_{k-1}, \hat{\theta}\right)}{\tilde{g}\left(u_{k-1}, \hat{\theta}\right)}
$$

with

$$
\begin{aligned}
\tilde{f}(\cdot)= & T_{p, k-1}\left(\hat{\theta}_{3}+\hat{\theta}_{4} u_{k-1}+\hat{\theta}_{5} u_{k-1}^{2}\right) \\
& +T_{e, k-1}\left(\hat{\theta}_{6}+\hat{\theta}_{7} u_{k-1}+\hat{\theta}_{8} u_{k-1}^{2}\right) \\
& +X_{e, k-1}\left(\hat{\theta}_{9}+\hat{\theta}_{10} u_{k-1}+\hat{\theta}_{11} u_{k-1}^{2}\right) \\
& +\hat{\theta}_{12} u_{k-1}+\hat{\theta}_{13} u_{k-1}^{2}+\hat{\theta}_{14} \\
\tilde{g}(\cdot)= & 1-\hat{\theta}_{1} u_{k-1}+\hat{\theta}_{2} u_{k-1}^{2}
\end{aligned}
$$

\section{Estimation}

In this section, different least-squares techniques are used to estimate parameters of the storage model (14).

\section{A. Nonlinear least-squares}

Depending on the type of problem an appropriate algorithm must be chosen to solve a nonlinear least squares problem [2], [3]. All methods have in common that the solution is iteratively found. Existence of local minima can be a serious issue (e.g. Fig. 1) if initial estimates are inaccurate. Hence, in practice, in particular in non-convex problems, most often the number of parameters to be estimated should be limited. It is therefor necessary to fi nd the most sensitive parameters. A (local) sensitivity analysis can be used to gain insight in the parameter sensitivity. Now, with knowledge of the sensitivities of the parameters, a set of parameters to be estimated is chosen. When physical and design parameters are to be estimated, usually these parameters may not vary unlimited. The least-squares problem then becomes constrained. However, this may lead to a non-optimal estimation in least-squares sense. Let us now demonstrate this to the storage model (14).

The parameter vector $p$ of the discrete-time storage model (14) consists of several design and physical parameters. The results of a sensitivity analysis are presented in Fig. 2. The parameters $c_{p p}, V_{p}$ and $\rho_{p}$ have the same sensitivity. They always appear product-wise and could be replaced by a combined parameter. Hence, only one of these parameters has to be estimated. Given the sensitivity in Fig. 2 and some indication of the uncertainty of the physical and design parameters the following parameters have been selected for estimation: $\hat{\theta}=\left[P_{\text {resp }}, \phi_{\text {max }}, c_{p p}, \alpha_{e a}, h\right]^{T}$, respectively the

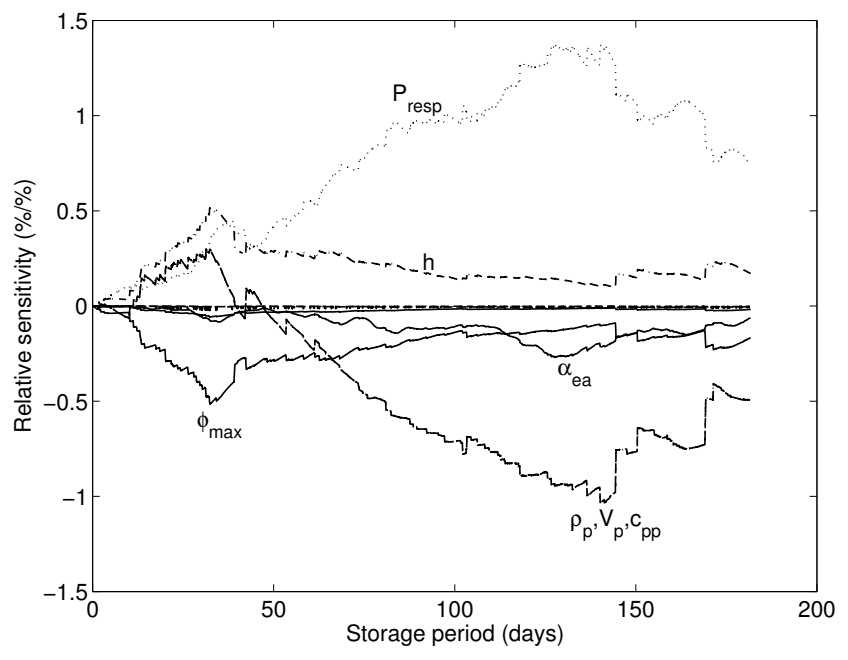

Fig. 2. Sensitivity of some design and physical parameters of a storage model.

TABLE I

NOMINAL AND ESTIMATED PARAMETER VALUES.

\begin{tabular}{ccc} 
& $\theta^{0}$ & $\hat{\theta}$ \\
\hline$P_{\text {resp }}$ & 5.42 & 5.17 \\
$\phi_{\text {max }}$ & 0.0972 & 0.0744 \\
$c_{p p}$ & 3600 & 4277 \\
$\alpha_{\text {ea }}$ & 0.347 & 0.347 \\
$h$ & 3.5 & 3
\end{tabular}

respiration heat of the stored product, maximum ventilation flow, the heat capacity of the stored product, heat transfer from environment to air in bulk and the height of the bulk. Subsequently, a constrained nonlinear least-squares estimation is performed. The nominal values $\left(\theta^{0}\right)$ and the estimates $(\hat{\theta})$ are given in Table I. Only the parameter estimate of $h$ is at its lower bound; the other parameter estimates are within the associated bounds.

\section{B. Truncated least-squares}

Given a model that is linear in its parameters, i.e.

$$
y=C \theta+e
$$

with $y \in \mathbb{R}^{N}, C \in \mathbb{R}^{N \times n}$ and $\theta \in \mathbb{R}^{n}$. An ordinary leastsquares estimate is given by

$$
\hat{\theta}=\left(C^{T} C\right)^{-1} C^{T} y
$$

However, when the problem is ill-conditioned the estimates are very sensitive to the data. To overcome this problem, the truncated least-squares method can be used. Given the singular value decomposition of $C$, i.e.

$$
C=U \Sigma V^{T}
$$

with $U \in \mathbb{R}^{N \times N}, \Sigma \in \mathbb{R}^{N \times n}$ and $V \in \mathbb{R}^{n \times n}$, one can determine from the singular values (diagonal elements of $\Sigma$ ) whether the problem is ill-conditioned. Premultiplying (17) 
with $U^{T}$ [2, p.77] gives:

$$
\begin{aligned}
y^{*} & =U^{T} y=U^{T} C \theta+U^{T} e \\
& =U^{T} U \Sigma V^{T} \theta+U^{T} e \\
& =\Sigma \theta^{*}+e^{*}
\end{aligned}
$$

where $\theta^{*}=V^{T} \theta$ and $e^{*}=U^{T} e$. The parameters $\theta_{i}^{*}$ that correspond to singular values that are very small compared to the largest singular value, defi ned by the numerical rank determinator $\mathcal{R}$, are set to 0 . Now, the modified linear regression model (20) can be solved. The sum of squares $\left(y^{*}-\Sigma \theta^{*}\right)^{T}\left(y^{*}-\Sigma \theta^{*}\right)$ is minimized when $\hat{\theta}_{i}^{*}=y_{i}^{*} / \sigma_{i}$ where $\sigma$ are the singular values and $i=1, \ldots, r$ with $r \leq n$ the numerical rank. The parameter estimates can now be obtained from $\hat{\theta}=V \hat{\theta}^{*}$.

Let us now analyze the storage model (15) and add the equation error $e_{k}$. Observations from a 50 days period with a sampling interval of 900 seconds are used to estimate the parameter vector $\theta$. Using the singular value decomposition (19) we obtain the matrices $U, V$ and $\Sigma$ related to the regression matrix of (15). By choosing an appropriate numerical rank determinator e.g. $\mathcal{R}=\bar{\sigma} / 1000$, with $\bar{\sigma}$ the largest singular value, the following results are obtained:

$$
S=\left[\begin{array}{c}
617.5027 \\
225.9039 \\
206.0795 \\
27.7489 \\
15.7058 \\
8.1518 \\
2.0180 \\
1.6773 \\
0.2375 \\
0.1451 \\
0.0270 \\
0.0246 \\
0.0019 \\
0.0003
\end{array}\right] \hat{\theta}^{*}=\left[\begin{array}{c}
-0.7604 \\
-0.3290 \\
-0.5414 \\
-0.0237 \\
-0.0196 \\
0.1382 \\
0.0052 \\
-0.0041 \\
0 \\
0 \\
0 \\
0 \\
0 \\
0
\end{array}\right]
$$

$$
\begin{aligned}
& \hat{\theta}=\left[\begin{array}{lllll}
-0.0038 & -0.0007 & 0.9998 & -0.0038
\end{array}\right. \\
& \begin{array}{llll}
0.0007 & 0.0001 & -0.0003 & 0.0043
\end{array} \\
& \begin{array}{llll}
-6.0 \cdot 10^{-5} & -2.9 \cdot 10^{-6} & 1.6 \cdot 10^{-6} & -0.0009
\end{array} \\
& \left.-4.6 \cdot 10^{-5} \quad 0.0024\right]^{T}
\end{aligned}
$$

From the right singular vectors (not shown here) it appears that the first singular value is predominantly related to $\theta_{3}$ and $\theta_{6}$ and the lowest singular value is related to $\theta_{10}$ and $\theta_{11}$.

If physical interpretation of the estimates is desired, it is possible to retain the original physical parameters from the estimated parameters using:

$$
\hat{\theta}_{i}=\varphi_{i}(\hat{p})
$$

where $\hat{\theta} \in \mathbb{R}^{n}$ is the estimated parameter vector and $\hat{p} \in \mathbb{R}^{q}$ the physical and/or design parameter vector to be estimated.

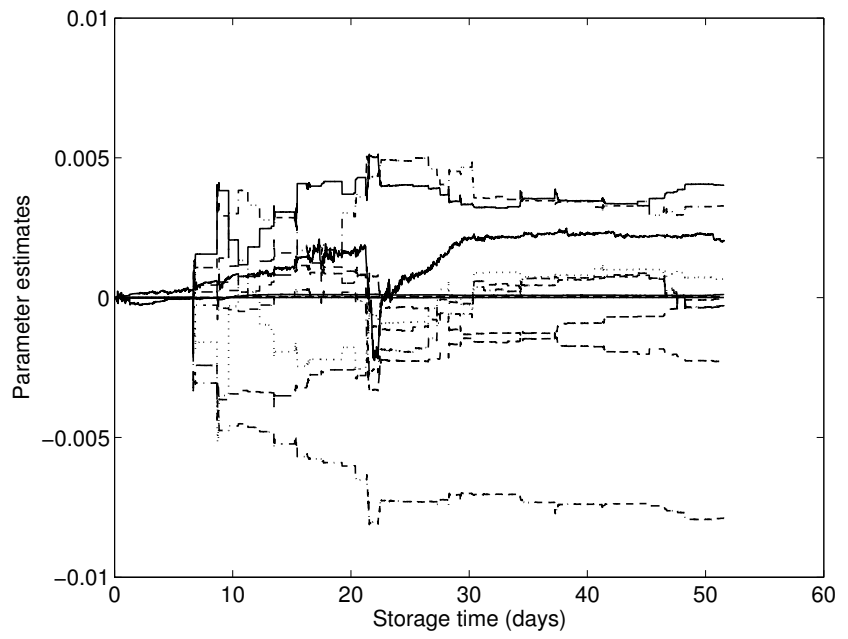

Fig. 3. Parameter evolution of the recursive parameter estimates $\theta_{1}, \theta_{2}$ and $\theta_{4} \ldots \theta_{14}$

If $q=n$ direct inversion could be used, while in other cases one should use e.g. a minimum length or least-squares approximation. However, and this should be stressed again, for prediction (16) can be directly applied.

\section{Recursive estimation}

A Kalman fi lter approach can be used to estimate parameters recursively [1]. The linear regression model of (17) should then be written in a form like

$$
\begin{aligned}
\theta(k+1) & =\theta(k)+w(k) \\
y(k) & =C(k) \theta(k)+v(k)
\end{aligned}
$$

Under the assumption that the parameters are constant the covariance matrix $E\left(w(k) w(k)^{T}\right)=0$. In addition to this, an assumption about the observation noise properties must be made and the initial estimate $\theta(0)$ and initial covariance matrix $P(0)$ must be specifi ed. The parameters of (15) are now estimated recursively.

Given a sampling time much smaller than the system timeconstant of (15) the initial parameter estimate of $\theta_{3}=1$ and all other initial parameter estimates are zero, i.e. the model reduces to $T_{p, k}=T_{p, k-1}$. The initial covariance matrix $P(0)=0.1 * I$, i.e. all parameters are considered to be independent and identically distributed with the knowledge that the actual parameter values are around the initial estimates. The covariance matrix, $R=E\left(v(k) v(k)^{T}\right)$, represents the variance of the observations, i.e. the product temperature, and determines the parameter update rate of the filter. In this example it is taken as $1^{\circ} C^{2}$. With given $y$ and $C$ from section III-B the parameter trajectories of $\theta_{1}, \theta_{2}$ and $\theta_{4} \ldots \theta_{14}$ are given in Fig. 3. Not shown is $\theta_{3}$ which remains close to one. From Fig. 3 it can be seen that it takes until day 8 before the parameters start to change. This is due to the fact that this is the fir rst ventilation period of the data-set. Furthermore, it can be seen that the parameters converge in time. The recursive 


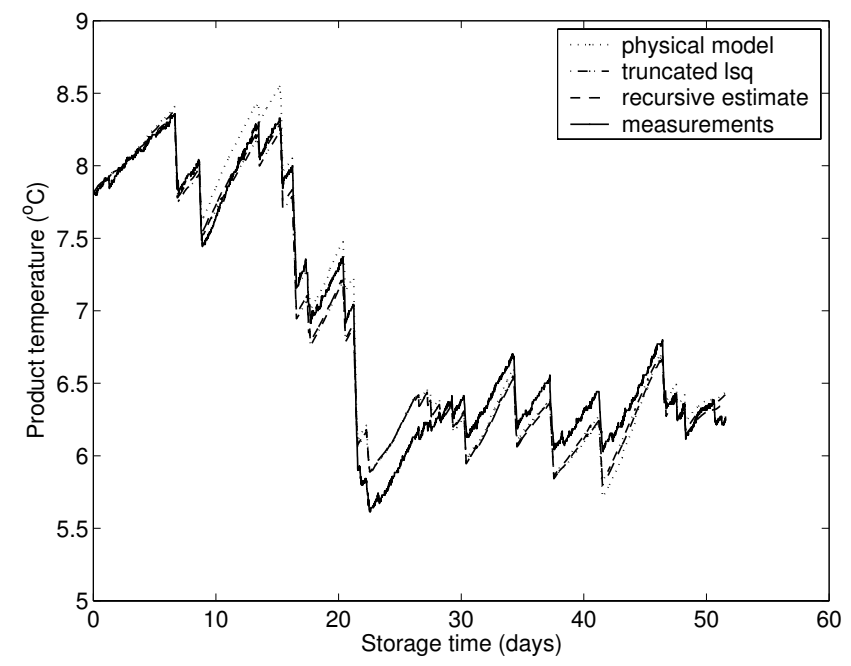

Fig. 4. Measured and predicted product temperatures in the calibration period

TABLE II

MEAN SQUARED ERRORS OF CALIBRATION AND VALIDATION PERIOD

\begin{tabular}{cccc} 
& physical model & truncated & recursive \\
\hline calibration & 0.027 & 0.020 & 0.021 \\
validation & 0.113 & 0.032 & 0.040
\end{tabular}

parameters estimates at the fi nal time are given by:

$$
\begin{gathered}
\hat{\theta}=\left[\begin{array}{lllll}
-4.5 \cdot 10^{-5} & 0.0040 & 0.9998 & -0.0079 \\
-0.0023 & 0.0001 & 0.0007 & 0.0033 & \\
3.9 \cdot 10^{-5} & 4.0 \cdot 10^{-6} & 4.4 \cdot 10^{-6} & -0.0003 \\
-0.0003 & 0.0021
\end{array}\right]^{T}
\end{gathered}
$$

This fi nal parameter vector at day 52 is used in the next section to test the validity of the recursively estimated model.

\section{VALIDATION}

In this section the estimated parameters from section III are validated by evaluating the model predictions obtained from an open loop simulation. First, the same period over which the calibration was performed is considered and then a validation period is chosen with about the same length.

First, the mean-square error (MSE) and a graphical presentation of the results of the calibration period are given in Table II and Fig. 4. It can be seen that the MSE of the recursive estimate and the truncated least-squares estimate are within the same range. The physical model performs slightly worse. Notice furthermore, that the sudden changes of the recursive estimates of Fig. 3 correspond to the fast dynamics, i.e. ventilation, of Fig. 4.

A validation step is performed over a different period in the same storage season. Hence, for the same storage facility and product, the same parameter vector as is obtained from calibration could be used. The results of the validation are given in Table II and Fig. 5. The model predictions with the truncated least-squares estimate and the recursive estimate

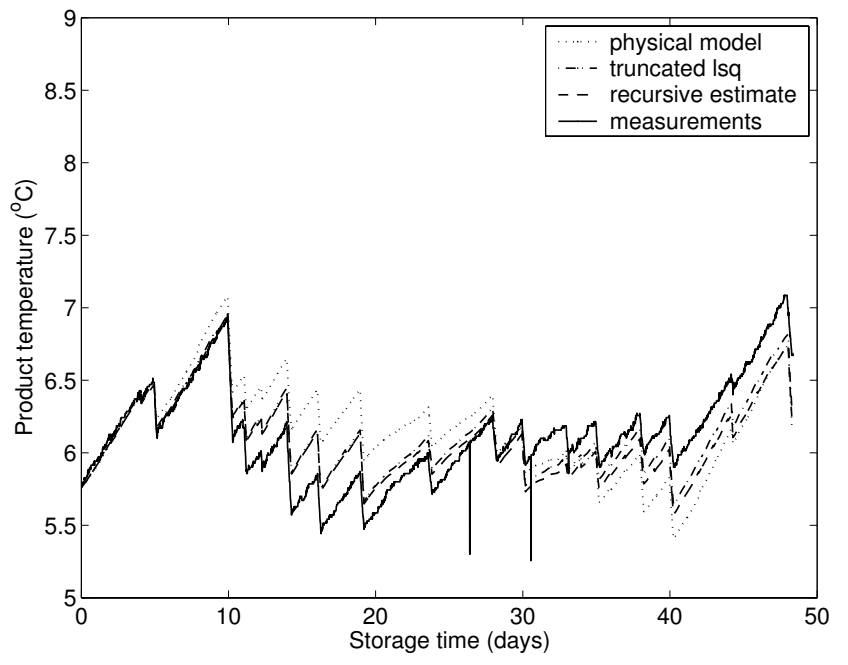

Fig. 5. Measured and predicted product temperatures in the validation period

perform within the same range. The physical model predicts signifi cantly worse.

\section{Discussion}

Reparameterizing and rearranging a discrete-time model with polynomial quotient structure into a model linear in its parameters is helpful in getting optimal parameter estimates in least-squares sense if the model is noise free. The physical interpretation of the variables is lost but the main structure is conserved. In contrast, black-box modelling on the basis of neural networks or nonlinear regressions can also be used. However, in those cases the system order must be estimated from the data. Usually, no direct information about the physical system is then taken into account.

Some diffi culties may arise in least-squares estimation of the reparameterized system. Rearranging and reparameterizing may lead to correlated columns and interdependent parameters, which lead to (near) rank defi cient problems. This problem can be solved by using truncated least-squares (see section III-B). But, the main problem is that due to the reparameterization there is not only (structural) unknown uncertainty in the output vector but also in the data matrix (see equation (5)). This problem can be tackled by a total least-squares approach [9] and will be subject of another paper.

In the storage model example the inputs, as external temperature $\left(T_{e}\right)$ and humidity $\left(X_{e}\right)$, are measured variables, which directly leads to an errors-in-variables problem. Consequently, a nonlinear estimation procedure should take this into account, as well. However, the key problem in this example was the numerical rank defi ciency of the data matrix. This problem not only occurred due to the introduction of correlated columns, but also due to the experimental setup. First, the fan is mainly controlled as on/off and it is switched on for less then $10 \%$ of the time. Therefor, the columns of $C$ that are multiplied by $u_{k-1}$ and $u_{k-1}^{2}$ will contain many zeros and as such will be highly correlated. In addition to this, the 
control frequency was quite high, i.e. 15 minutes compared to the time constant of the system which is approximately 19 hours with maximum ventilation and 20 days with no ventilation, where the optimal sample time is about $1 / 10$ of the time constant [1], and thus leading to high correlations.

Truncated least-squares came out quite well in the example. However, an appropriate value for the numerical rank determinator must be chosen. Here it is chosen by tuning. The nonlinear least-squares estimation performs worse for both calibration as well as for validation. From Table I it can be seen that the parameter $h$ is bounded at its lower bound, which indicates that the estimate is non-optimal. Furthermore, the solution can be in a local minimum. The recursive estimate performs well for both calibration and validation period.

\section{CONCLUDING REMARKS}

It has been demonstrated that a discrete-time model with polynomial quotient structure in input, output, and parameters can be rearranged and reparameterized such that a model arises that is linear in its parameters. Consequently, the parameters can be uniquely estimated by ordinary leastsquares methods. Furthermore, it has been shown that using the estimated parameters in the back-transformed model (via rearranging terms) leads to a predictor with fairly good open loop predictive performance in the sense of mean-square error. Further research will focus on a total least-squares approach to account for the errors-in-variables problem.

\section{ACKNOWLEDGEMENTS}

We are indebted to Tolsma for providing the data. This research is part of the WIC2 project, which is carried out in co-operation with PRIVA Hortimation, Tolsma, WNI and Agrotechnology and Food Innovations.

\section{REFERENCES}

[1] L. Ljung, System Identification, Theory for the User, ser. Information and System Sciences. Englewood Cliffs, New Jersey: Prentice Hall, Inc., 1987.

[2] J. P. Norton, An Introduction to Identification. London: Academic Press, 1986.

[3] J. E. Dennis and R. B. Schnabel, Numerical Methods for Unconstrained Optimization and Nonlinear Equations, ser. Classics in Applied Mathematics. Englewood Cliffs, NJ: Prentice Hall, Inc, 1996, vol. 16.

[4] A. Gelb, Applied Optimal Estimation. Cambridge, Massachusetts: MIT Press, 1974.

[5] L. Ljung and T. Glad, "On global identifi ability for arbitrary model parametrizations," Automatica, vol. 30(2), pp. 265-276, 1994.

[6] L. J. S. Lukasse, K. J. Keesman, and G. van Straten, "Grey-Box Identifi cation of Dissolved Oxygen Dynamics in an Activated Sludge Process," in Proc. 13th IFAC world congress, San Francisco, USA, Vol. $N, 1996$, pp. 485-490.

[7] T. Kailath, Linear systems, ser. Prentice-Hall Information and System Sciences Series. Englewood cliffs, New Jersey: Prentice-Hall, 1980.

[8] K. J. Keesman, D. Peters, and L. J. S. Lukasse, "Optimal climate control of a storage facility using local weather forecasts," Control Engineering Practice, vol. 11, no. 5, pp. 505-516, 2003.

[9] G. H. Golub and C. F. Van Loan, "An analysis of the total least squares problem," SIAM Journal on Numerical Analysis, vol. 17, no. 6, pp. 883-893, 1980 . 